\title{
Detección de hipertensión arterial y su relación con otros factores de riesgo cardiovascular en adolescentes que concurren a realizar el apto físico escolar
} Detection of hypertension and its relation to other cardiovascular risk factors in
adolescents who request a medical fitness certificate for school

Dra. Micaela Amor ${ }^{a}$ Dra. Luciana Pronskya , Dra. Carla Irazabal ${ }^{a}$ Dra. Sofía Ortiz de Latierro ${ }^{a}$ Dra. María B. Tapia ${ }^{a}$ y Dra. Eugenia Sarcona ${ }^{a}$

\section{RESUMEN}

La hipertensión arterial (HTA) puede comenzar en la niñez y se relaciona con otros factores de riesgo, como la obesidad y el sedentarismo.

En este estudio, se estimó la prevalencia y se evaluaron los factores asociados a HTA en adolescentes de entre 15 y 19 años que concurrieron al Centro de Salud y Acción Comunitaria $\mathrm{N}^{\circ} 12$ para realizar el apto físico escolar. Se obtuvieron los siguientes datos: edad, sexo, antecedentes familiares de HTA, tabaquismo actual, actividad física, peso, talla, perímetro de cintura y presión arterial.

Se evaluaron 289 adolescentes con una edad media de 16,6 años; el 48,6 \% fueron varones. El 6,6 \% presentó HTA; el 10,9\%, obesidad, y el 13,4 \%, perímetro de cintura aumentado. La presencia deHTA se asoció significativamente a la presencia de obesidad y perímetro de cintura aumentado, y no se encontró asociación significativa con las demás variables estudiadas. Palabras clave: adolescente, hipertensión arterial, estilo de vida sedentario, obesidad, aptitud física.

http:/ / dx.doi.org/10.5546/ aap.2019.344

Texto completo en inglés:

http:/ / dx.doi.org/10.5546/ aap.2019.eng.344

Cómo citar: Amor M, Pronsky L, Irazabal C, Ortiz de Latierro S, et al. Detección de hipertensión arterial y su relación con otros factores de riesgo cardiovascular en adolescentes que concurren a realizar el apto físico escolar. Arch Argent Pediatr 2019;117(5):344-347.

a. Centro de Salud y Acción Comunitaria N. ${ }^{\circ}$ 12, Área Programática Hospital "I. Pirovano", Ciudad Autónoma de Buenos Aires.

Correspondencia:

Dra. Eugenia Sarcona: ugesarcona@hotmail.com

Financiamiento: Ninguno.

Conflicto de intereses: Ninguno que declarar.

Recibido: 22-10-2018

Aceptado: 16-4-2019

\section{INTRODUCCIÓN}

La enfermedad cardiovascular continúa siendo la causa más común de discapacidad y muerte y la segunda causa de insuficiencia renal crónica en adultos, tanto en nuestro país como a nivel mundial. ${ }^{1,2}$ En muchas oportunidades, esto puede prevenirse y un adecuado control de la presión arterial (PA) y de otros factores de riesgo (FR) desde la infancia disminuye la incidencia de enfermedad cardiovascular en la adultez. ${ }^{3}$

La pandemia de obesidad en los niños y en los adolescentes, junto con el riesgo de desarrollar tempranamente hipertrofia ventricular y aterosclerosis, vuelve importante el rastreo de FR cardiovascular desde la niñez para reducir riesgos de salud a largo plazo. La HTA esencial es la principal causa de HTA en los adolescentes y tiene múltiples FR asociados; la obesidad es el que más prevalece. ${ }^{4}$

La información acerca de la prevalencia de HTA en los adolescentes sanos es escasa a nivel mundial y en nuestro medio en particular, con cifras que varían entre el $2 \%$ y el $8 \%$ en la población menor de 18 años.,

Teniendo en cuenta que el nivel de PA en la adolescencia es el principal FR para el desarrollo de HTA en los adultos, ${ }^{7}$ reviste interés conocer la prevalencia de la HTA en este grupo etario y su relación con otros FR cardiovascular a fin de instaurar precozmente acciones preventivas y de promoción de la salud.

\section{OBJETIVO}

Estimar la prevalencia y evaluar los factores asociados a HTA en los adolescentes que concurren a realizar el apto físico escolar (AFE).

\section{MATERIALES Y MÉTODOS}

Diseño: Estudio analítico y transversal.

Criterios de inclusión: Adolescentes de entre 
15 y 19 años inclusive que asistieron al Centro de Salud y Acción Comunitaria N ${ }^{\circ} 12$ (CeSAC 12), dependiente del Hospital Ignacio Pirovano de la Ciudad Autónoma de Buenos Aires, a realizar el AFE entre los meses de mayo y octubre de 2017, y que aceptaron participar del estudio.

Criterios de exclusión: Pacientes con HTA medicados, HTA secundaria diagnosticada, diabéticos, con antecedentes de enfermedad renal o cardiovascular y con antecedentes de enfermedades reumáticas juveniles.

\section{Variables:}

Presión arterial

- HTA: promedio de la PA sistólica y/o diastólica $\geq$ al percentil 95.

- Presión arterial elevada: promedio de la PA sistólica y / o diastólica $\geq$ al percentil $90 \mathrm{y}<\mathrm{al}$ percentil 95.

- Normotensión: promedio de la PA sistólica y/o diastólica $<$ al percentil 90.

Medición de la PA: Se realizó con el paciente sentado, la espalda apoyada, el brazo dominante apoyado a la altura del corazón y luego de 30 minutos de no haber fumado o realizado actividad física (AF). Se utilizó el valor promedio de tres mediciones cada 2 minutos con tensiómetro semiautomático Omron HEM-741 CINT.

Índice de masa corporal (IMC): Se calculó como el peso $(\mathrm{kg}) /$ talla $\left(\mathrm{m}^{2}\right)$. Se utilizó el percentil de IMC $>85$ para definir sobrepeso y $>97$ para definir obesidad. ${ }^{8}$

Circunferencia de la cintura: Perímetro medido a la altura del borde superior de la cresta ilíaca con cinta métrica inextensible Calibres Argentinos. Se obtuvo con el individuo de pie, con la cintura descubierta, los brazos relajados al costado del cuerpo y con respiración tranquila. Se consideró aumentada $>$ al percentil $90 .{ }^{8}$

AF: Se midió como la cantidad de minutos diarios dedicados a la AF. Se realizó la sumatoria de los minutos que se dedicaban a la AF cada uno de los días de la semana y se dividió por 7 para obtener el promedio de los minutos diarios. Se consideró sedentario a todo aquel individuo que hubiere realizado menos de 60 minutos diarios de AF. ${ }^{9}$

Antecedentes familiares de HTA: Se consideraron positivos en aquellos adolescentes que refirieron tener padre y/o madre con HTA.

Tabaquismo: Se consideró positivo el tabaquismo actual.

Sexo: Sexo biológico.
Edad: Años y meses cumplidos. Análisis estadístico:

Se evaluó al adolescente según el sexo y la talla utilizando las tablas habituales de la Sociedad Argentina de Pediatría para, posteriormente, ubicar, según la edad y el percentil de talla, en las tablas de PA de la Academia Estadounidense de Pediatría. ${ }^{10,11}$

Para la descripción, se utilizaron números absolutos y porcentajes o media y desvío estándar según correspondiera. Para el análisis uni- y multivariado, se utilizaron las pruebas de chi ${ }^{2}$ o la prueba exacta de Fisher y la regresión logística. Se consideró una significación estadística $\mathrm{p}<0,05$ e intervalos de confianza (IC) del $95 \%$. Se utilizaron los programas estadísticos Statistix versión 8.0 y VCCstat v.Beta 2.11.

\section{Consideraciones éticas}

Este estudio fue aprobado por el Comité de Ética en Investigación del Hospital I. Pirovano y, en todos los casos, se obtuvo el consentimiento informado del participante por escrito.

A todos los adolescentes y a sus familiares a los que se les encontró algún FR se los asesoró acerca de este y se los volvió a citar para confirmar el diagnóstico y realizar el seguimiento.

\section{RESULTADOS}

Se evaluaron 289 adolescentes de entre 15 y 19 años de edad. Se excluyó una paciente por encontrarse con medicación antihipertensiva. Todos los demás pacientes que concurrieron al CeSAC 12 en el marco del AFE aceptaron participar del estudio y fueron elegibles.

Edad media: 16,6; 134 (el 46,4\%) varones. 19 (el 6,6 \%) presentaron HTA; 66 (el 22,8 \%), PA elevada; 33 (el 11,4\%), obesidad; 41 (el 14,2\%), sobrepeso; 42 (el 14,5\%), perímetro de cintura aumentado (PCA); 63 (el 21,8\%), antecedentes familiares de HTA; 33 (el 11,4\%), tabaquismo; 232 (el 80,3\%), sedentarismo (Tabla 1).

En el análisis univariado, se encontró una asociación significativa entre los valores de HTA y el sexo masculino ( $\mathrm{p}<0,001$, odds ratio $-O R-$ 6,9 , IC $95 \%$ : 2-23,9), la obesidad y el sobrepeso $(\mathrm{p}<0,001$, OR 5,7, IC $95 \%: 2,2-15,2)$ y el PCA $(\mathrm{p}<0,001$, OR 8,3, IC $95 \%$ : 3,1-21,9) (Tabla 2).

En el análisis multivariado ajustado por sexo, la obesidad, estimada tanto por IMC como por PCA, continuó mostrando una relación significativa e independiente con la presencia de HTA (PCA p < 0,001, OR 10,2, IC $95 \%$ : 3,6-28,8; obesidad y sobrepeso $\mathrm{p}<0,001$, OR 7,3, IC $95 \%$ : 
2,6-20,0; obesidad $\mathrm{p}<0,001$, OR 11,8, IC $95 \%$ : 4,034,7) (Tabla 3).

\section{DISCUSIÓN}

Se encontró, en los adolescentes estudiados, una prevalencia de HTA del 6,6\%, asociada, principalmente, a la obesidad y al sobrepeso, cuyas prevalencias fueron del $11,4 \%$ y del $14,2 \%$, respectivamente.

El estudio de los factores de riesgo cardiovascular en adolescentes (ERICA) ${ }^{5}$ sugirió que la enfermedad cardiovascular tenía su origen en edades tempranas de la vida y que el riesgo de desarrollarla estaba determinado por el efecto

TABla 1. Características generales. $N=289$

\begin{tabular}{|c|c|c|c|}
\hline & & $\%(\mathrm{n})$ & IC $95 \%$ \\
\hline \multirow[t]{6}{*}{ Edad (años) (media $\pm \mathrm{DE})$} & & $16,6 \pm 1,29$ & \\
\hline & 15 & $22,5(65)$ & \\
\hline & 16 & $28(81)$ & \\
\hline & 17 & $23,9(69)$ & \\
\hline & 18 & $13,8(40)$ & \\
\hline & 19 & $11,8(34)$ & \\
\hline \multirow[t]{2}{*}{ Sexo } & Mujeres & $53,6(155)$ & \\
\hline & Varones & $46,4(134)$ & \\
\hline Antecedentes familiares de HTA & & $21,8(63)$ & $17,3-27,1$ \\
\hline Tabaquismo actual & & $11,4(33)$ & $8,1-15,8$ \\
\hline Sedentarios & & $80,3(232)$ & $75,1-84,6$ \\
\hline Cintura aumentada & & $14,5(42)$ & $10,8-19,2$ \\
\hline \multirow[t]{2}{*}{ Estado nutricional } & Sobrepeso & $14,2(41)$ & $10,5-18,9$ \\
\hline & Obesidad & $11,4(33)$ & $8,1-15,8$ \\
\hline Presión arterial elevada & & $22,8(66)$ & $18,2-28,2$ \\
\hline HTA & & $6,6(19)$ & $4,1-10,2$ \\
\hline
\end{tabular}

IC: intervalo de confianza; DE: desvío estándar; HTA: hipertensión arterial.

TABla 2. Análisis univariado. $N=289$

\begin{tabular}{|c|c|c|c|c|c|c|}
\hline & Todos & HTA, n (\%) & No HTA, n (\%) & $p$ & OR & IC $95 \%$ \\
\hline Varones (b) & $134(46,4)$ & $16(5,5)$ & $118(40,8)$ & $<0,001$ & 6,9 & $2-23,9$ \\
\hline Sedentarios (b) & $232(80,3)$ & $14(4,8)$ & $218(75,4)$ & 0,46 & 0,7 & $0,2-1,9$ \\
\hline Antecedentes familiares (a) & $63(21,8)$ & $5(1,7)$ & $58(20,1)$ & 0,62 & 1,3 & $0,4-3,8$ \\
\hline Tabaquistas (a) & $33(11,4)$ & $2(0,7)$ & $31(10,7)$ & 0,9 & 0,9 & $0,2-4,1$ \\
\hline Cintura aumentada (b) & $42(14,5)$ & $10(3,5)$ & $32(11,1)$ & $<0,001$ & 8,3 & $3,1-21,9$ \\
\hline Obesidad y sobrepeso (b) & $74(28,7)$ & $12(4,1)$ & $62(21,4)$ & $<0,001$ & 5,7 & $2,2-15,2$ \\
\hline Obesos (b) & $33(11,4)$ & $9(3,1)$ & $24(8,3)$ & $<0,001$ & 9,2 & $3,4-24,9$ \\
\hline
\end{tabular}

IC: intervalo de confianza; OR: odds ratio; HTA: hipertensión arterial.

(a) prueba exacta de Fisher, (b) prueba de $\mathrm{chi}^{2}$, valor de $\mathrm{p}=0,05$.

TABla 3. Análisis multivariado. $N=289$

\begin{tabular}{|c|c|c|c|c|c|}
\hline & Coeficiente & Error estándar & $p$ & OR & IC $95 \%$ \\
\hline Sexo & 2,16 & 0,66 & 0,001 & 8,7 & $2,4-31$ \\
\hline Obesidad y sobrepeso & 1,98 & 0,51 & $<0,001$ & 7,3 & $2,6-20$ \\
\hline Obesidad & 2,46 & 0,55 & $<0,001$ & 11,8 & $4,0-34,7$ \\
\hline Cintura aumentada & 2,32 & 0,53 & $<0,001$ & 10,2 & $3,6-28,8$ \\
\hline
\end{tabular}

OR: odds ratio; IC: intervalo de confianza.

Regresión logística; se ajustó la presencia de obesidad y sobrepeso, obesidad y cintura aumentada por sexo masculino, en relación con la HTA. Valor de $p=0,05$. 
sinérgico de los FR cardiovascular a través del tiempo. La asociación frecuente de obesidad, sedentarismo, dislipemia y diabetes aumentó el poder aterogénico de la HTA.

En otro estudio, ${ }^{12}$ se determinó que los adolescentes con sobrepeso y obesidad presentaron mayor adiposidad corporal y PA que sus similares con peso normal. A su vez, se observó una asociación entre el estado nutricional y la PA en ambos sexos.

El aumento de la obesidad, estimada tanto por IMC como por perímetro de cintura, mostró una estrecha relación con valores aumentados de PA en nuestro estudio y en estudios similares realizados en diferentes lugares de nuestro país y desde edades aún más tempranas al comienzo de la adolescencia. ${ }^{13-15}$

No se encontró una relación significativa entre los valores elevados de PA y los FR, como antecedentes familiares, sedentarismo y tabaquismo. Se cree que esto se explica por el tamaño reducido de la muestra, principal limitación de este trabajo. Llama la atención el alto porcentaje de sedentarismo (el 80,3\%). Se considera que se debe, en parte, al parámetro elegido para estimarlo (una hora de AF diaria) y al hecho de que a los adolescentes estudiados no se les permitía realizar AF en la escuela hasta la obtención del AFE, momento de la realización del estudio. Así, la AF medida fue la extraescolar, lo que, probablemente, aumentara la prevalencia de sedentarismo.

Por otra parte, en este estudio, se midió la PA en el brazo dominante y no siempre en el brazo derecho, lo que pudo llevar a subdiagnosticar HTA en los pacientes zurdos con coartación aórtica. Se espera que esta subestimación haya quedado minimizada dada la edad de los participantes del estudio, la baja prevalencia de coartación aórtica y el hecho de que la mayor parte de la población era diestra.

Cabe destacar la importancia y obligatoriedad de medir la PA y evaluar el estado nutricional en las consultas con los adolescentes. Teniendo en cuenta los valores obtenidos, se debe efectuar un plan de acción que incluya la asesoría en alimentación saludable y subraye la importancia de realizar ejercicio de tipo aeróbico como medida preventiva.

La consulta por el AFE solicitado por las escuelas, muchas veces, es la única oportunidad de contacto de este grupo etario con el sistema de salud, motivo por el cual el AFE se convierte, así, en una instancia de educación y empoderamiento en hábitos y prácticas saludables del adolescente en preparación para el resto de su vida adulta.

En conclusión, la presencia de HTA en la población adolescente estudiada fue del $6,6 \%$ y se asoció a la presencia de obesidad y PCA, y no se encontró una asociación significativa con las demás variables estudiadas.

\section{REFERENCIAS}

1. Organización Mundial de la Salud. Las 10 principales causas de defunción. [Acceso: marzo de 2019]. Disponible en https: / / www.who.int/es/news-room/fact-sheets / detail/the-top-10-causes-of-death.

2. Argentina. Ministerio de Salud. Dirección Nacional de promoción de la salud y control de enfermedades no transmisibles. Mortalidad. [Acceso: abril de 2019]. Disponible en http: / / www.msal.gob.ar/ent/index.php/ vigilancia/ areas-de-vigilancia/mortalidad.

3. Assadi F. Prehypertension: a warning sign of future cardiovascular risk. Int J Prev Med. 2014; 5(Suppl 1):S4-9.

4. Yang Q, Zhong Y, Merritt R, Coqswell ME. Trends in High Blood Pressure among United States Adolescents across Body Weight Category between 1988 and 2012. J Pediatr. 2016; 169:166-73.e3.

5. Abraham CW, Blanco G, Coloma G, Cristaldi A, et al. ERICA. Estudio de los factores de riesgo cardiovascular en adolescentes. Rev Fed Arg Cardiol. 2013; 42(1):29-34.

6. Díaz A, Calandra L. Presión arterial elevada en niños y adolescentes escolarizados de Argentina en los últimos 25 años: revisión sistemática de estudios observacionales. Arch Argent Pediatr. 2017; 115(1):5-11.

7. Redwine K, Falkner B. Progression of Prehypertension to Hypertension in Adolescents. Curr Hypertens Rep. 2012; 14(6):619-25.

8. Comité Nacional de Nutrición, Sociedad Argentina de Pediatría. Guías de práctica clínica para la prevención, el diagnóstico y el tratamiento de la obesidad. Arch Argent Pediatr. 2011; 109(3):256-66.

9. Organización Mundial de la Salud. Recomendaciones mundiales sobre actividad física para la salud. 2010. [Acceso: abril de 2019]. Disponible en: https: / / www.who. int/dietphysicalactivity/factsheet_recommendations/es/.

10. National High Blood Pressure Education Program Working Group on High Blood Pressure in Children and Adolescents. The Fourth Report on the Diagnosis, Evaluation, and Treatment of High Blood Pressure in Children and Adolescents. Pediatrics. 2004; 114(2 suppl, 4th Report):555-76.

11. Grupo de Hipertensión, Sociedad Argentina de Pediatría. Consenso sobre factores de enfermedad cardiovascular en pediatría. Hipertensión arterial en el niño y el adolescente. Arch Argent Pediatr. 2005; 103(4):348-66.

12. Cossio-Bolaños M, Cossio-Bolaños W, Araya Menacho A, Gómez Campos R, et al. Estado nutricional y presión arterial de adolescentes escolares. Arch Argent Pediatr. 2014; 112(4):302-7.

13. Gotthelf S, Fonseca M. Hipertensión arterial y su asociación con variables antropométricas en adolescentes escolarizados de la ciudad de Salta (Argentina). Rev Fed Arg Cardiol. 2012; 41(2):96-102.

14. Zeberio N, Malpeli A, Apezteguia M, Carballo M, et al. El estado nutricional de niños escolares y su relación con la tensión arterial. Arch Argent Pediatr. 2013; 111(2):92-7.

15. Szer G, Kovalsky I, De Gregorio M. Prevalencia de sobrepeso, obesidad y su relación con hipertensión arterial y centralización del tejido adiposo en escolares. Arch Argent Pediatr. 2010; 108(6):492-8. 